\title{
QoE-aware Energy Efficiency Maximization Based J oint User Access Selection and Power Allocation for Heterogeneous Network
}

\author{
Shiyu $\mathrm{Ji}^{1}$, Liangrui Tang ${ }^{1}$, Chen $\mathrm{Xu}^{1}$, Shimo $\mathrm{Du}^{2}$, Jiajia $\mathrm{Zhu}^{1}$, and Hailin $\mathrm{Hu}^{1}$, \\ ${ }^{1}$ State Key Laboratory of Alternate Electrical Power System, North China Electric Power University \\ Beijing, 102206 - China \\ [e-mail:15210726562@163.com] \\ ${ }^{2}$ China Mobile Company \\ Hangzhou, 310006- China \\ [e-mail: dushimo@163.com] \\ *Corresponding author: Shiyu Ji
}

Received March 11, 2017; revised May 9, 2017; accepted June 17, 2017;

published October 31, 2017

\begin{abstract}
In future, since the user experience plays a more and more important role in the development of today's communication systems, quality of experience (QoE) becomes a widely used metric, which reflects the subjective experience of end users for wireless service. In addition, the energy efficiency is an increasingly important problem with the explosive growth in the amount of wireless terminals and nodes. Hence, a QoE-aware energy efficiency maximization based joint user access selection and power allocation approach is proposed to solve the problem. We transform the joint allocation process to an optimization of energy efficiency by establishing an energy efficiency model, and then the optimization problem is solved by chaotic clone immune algorithm (CCIA). Numerical simulation results indicate that the proposed algorithm can efficiently and reliably improve the QoE and ensure high energy efficiency of networks.
\end{abstract}

Keywords: QoE-aware energy efficiency, joint allocation, heterogeneous networks, chaotic clone immune algorithm

A preliminary version of this paper appeared in ICNC-FSKD 2016, Aug. Changsha, China. This version main focus on the QoE of each users and establish the different QoE model, moreover, a plenty of implementation results on QoE is analzed in this version. This research was supported by the National High Technology Research and Development of China 863 Program (2014AA01A701) and Beijing Natural Science Foundation (4142049) and the Fundamental Research Funds for the Central Universities of China (2015XS07). 


\section{Introduction}

In future, a wide variety of Radio Access Technologies (RATs) including Wireless Local Area Network (WLAN) and Worldwide Interoperability for Microwave Access (WiMAX) are expected to be integrated to transmit different service [1,2].

The paradigm shift towards heterogeneity brings many new challenges to wireless network design. In future wireless network, a wide range of services can be offered to mobile users and the capacity of today's network is far from satisfaction, which will finally affect the QoE for users. Thus, one of the main challenges is that an efficient resource allocation strategy should be proposed to improve the QoE of users with limited resource utilization [3,4]. On the other hand, the macro-cell, microcell and femtocell network should be ultra-dense because of the huge traffic load in future which will cause lots of problems (e.g. high cost and pollution problem). Considering these financial and environmental problems, it is also necessary to improve the energy efficiency $[5,6]$.

Both of the QoE and the energy efficiency improvement are challenging tasks for resource management in heterogeneous networks. The authors in $[7,8]$ prove that the resource allocation can be essential and efficient to enhance user experience and energy efficiency.

\subsection{Motivation and Related Work}

Nowadays, a substantial amount of works focuses on the areas of resource allocation to improve the user experience and energy efficiency. These approaches can be divided into two classifications, the single resource allocation and the joint resource allocation.

The single resource allocation usually refers to user access selection [9,10], power control $[11,12]$ and spectrum sharing $[13,14]$. The authors in [9] investigate the users assigned to improve the utility of users. In [11], the power control algorithm is proposed to improve the energy efficiency. Although the single resource allocation perform well in providing the perfect objective metrics, it would not realize the maximum utilization of resource because of the relevance among different multi-dimensional resources.

Thus, much works have turned their focus from single resource allocation to joint resource allocation. Bandwidth allocation algorithm (TMBA) is proposed in [15]. In this work, the authors investigate the fundamental characteristics of user QoS and establish the throughput model of user with different type service. The authors in [16] propose a QoS-aware admission control with bandwidth borrow-return strategy to allow efficiently the traffic offloading from macro-cell to WLAN considering the user mobility and QoS constraints under fraction frequency reuse condition, and some works have investigated the spectrum sharing in heterogeneous networks. The authors in [17] investigate the comprehensive spectrum sharing considering multiple criteria, such as interference, cell load, and capacity in an unlicensed band based on software-defined network (SDN). The network selection and channel allocation for spectrum sharing is investigated in [18]. However, the authors in these literatures fail to consider the energy consumption. Since the energy consumption is out of consideration in most previous work, some joint allocation models [19-21] for energy consumption is put forward in recent years. However, the relationship between energy consumption and throughput is not considered in the algorithm.

To define the relationship between the energy consumption and throughput, the energy efficiency is proposed [22-25], where many methods are used to realize the energy efficiency improving [26-32]. In [26], energy efficiency scheme considering the circuit power 
consumption in orthogonal frequency division multiple access is investigated and the authors in [27] propose resource allocation is an efficiency method for energy efficiency improvement. The authors in [28] propose an energy efficiency maximization based joint power and bandwidth allocation (EEBA) within the QoS constraint to maximize the energy efficiency. In [29], the authors investigate the energy efficient resource allocation in multiple radio access technologies, however, the energy consumption in user side is ignored in this works. The energy efficiency considering the user side and network side is proposed in [30], which is in the perspective of operators and ignore that the users would also like to pursue the energy efficiency, so we establish the energy efficiency from users' perspective. However, theses algorithm pursue the energy efficiency improvement but ignore to promote the QoS degree of each service. Therefore, the authors in [33-35] propose a new performance metric, quality-aware energy efficiency to capture energy efficiency from service quality's perspective. Although QoS parameters perform well in providing a perfect objective metric, they could not directly reflect the perceived quality by wireless users, thus, the users would not satisfy the network. On the other hand, QoE can directly reflect the user satisfaction [36], Therefore, QoE-driven techniques can adaptively allocate the resources directly aiming at enhancing the perceived quality by wireless users.

Actually, there are some attempts on QoE-oriented resource allocation [37].The gradual resource allocation algorithm is proposed in [38], which aims to address the energy efficiency while guarantee the perceived QoE level of users. The authors in [39] aim to improve the QoE levels for all users rather than guarantee the QoE level. However, these literatures ignore the difference of QoE function for different type of users. Therefore, in this paper, we propose the QoE-aware energy efficiency based joint allocation algorithm (QEEBA). We formulate the QoE function for different type users and the energy efficiency of each users. In addition, the CCIA is proposed to optimize the QoE-aware energy efficiency to improve the QoE and energy efficiency.

\subsection{Contributions and Organization}

In this work, our aim is to find the optimal joint allocation method to maximize the QoE-aware energy efficiency. Thus, our contributions in this paper can be summarized as follows:

Firstly, we investigate the tradeoff of QoE and energy efficiency in user-centric perspective, and we formulate the different QoE function for different type users considering the difference feature of user experience. To guarantee the formulated QoE function can be used in dynamic real-time wireless system, we describe the QoE model as the function of the transmission rate.

Secondly, a joint user access selection and power allocation policy based on QoE-aware energy efficiency in heterogeneous networks is explored and translate the joint allocation problem to an optimization problem with constraints. Our optimization problem formulation also takes into account the circuit power consumption for joint allocation which consider the users and cells energy consumption.

Thirdly, we use the CCIA to solve the optimization problem with the QoS constraints and compared with the IA based joint allocation.

The remainder of this paper is organized as follows. In Section 2, we introduce the system model and the problem formulation is presented in Section 3. In Section 4, we describe the joint access selection and power allocation algorithm. Section 5 presents simulation experiments and analyzes the performances of joint allocation in heterogeneous networks. Finally, we draw the conclusion in Section 6. 


\section{System model}

As shown in Fig. 1, we consider the general case of heterogeneous network consisting the macro-cells whose set can be denoted as $\mathcal{M}_{1}=\left\{1,2, \ldots, M_{1}\right\}$ and the co-channel low power nodes (LPNs) whose set can be denoted as $\mathcal{M}_{2}=\left\{M_{1}+1, M_{1}+2, \ldots, M_{1}+M_{2}\right\}$, respectively. Furthermore, all network nodes can be denoted as $\mathcal{M}_{=} \mathcal{M}_{\mathbf{1}} \cup \mathcal{M}_{2}$. We also define $\mathcal{N}=\{1,2, \ldots, n, \ldots, N\}$ and $\mathcal{K}=\{1,2, \ldots, k, \ldots, K\}$ as the set of users and sub-channels, respectively. All users are randomly distributed in the heterogeneous network and the bandwidth of sub-channel is $B$. If the $n$th user accesses to the $m$ th network in channel $k$, $a_{m n}^{k}=\mathbf{1}$, otherwise, $a_{m n}^{k}=\mathbf{0}$. We denote that the $c_{n m}=\mathbf{1}$ means the $n$th user accesses to the $m$ th network, and $c_{m \boldsymbol{n}}=\mathbf{0}$ is not. We also assume that the users would use different application, which would transmit different service.

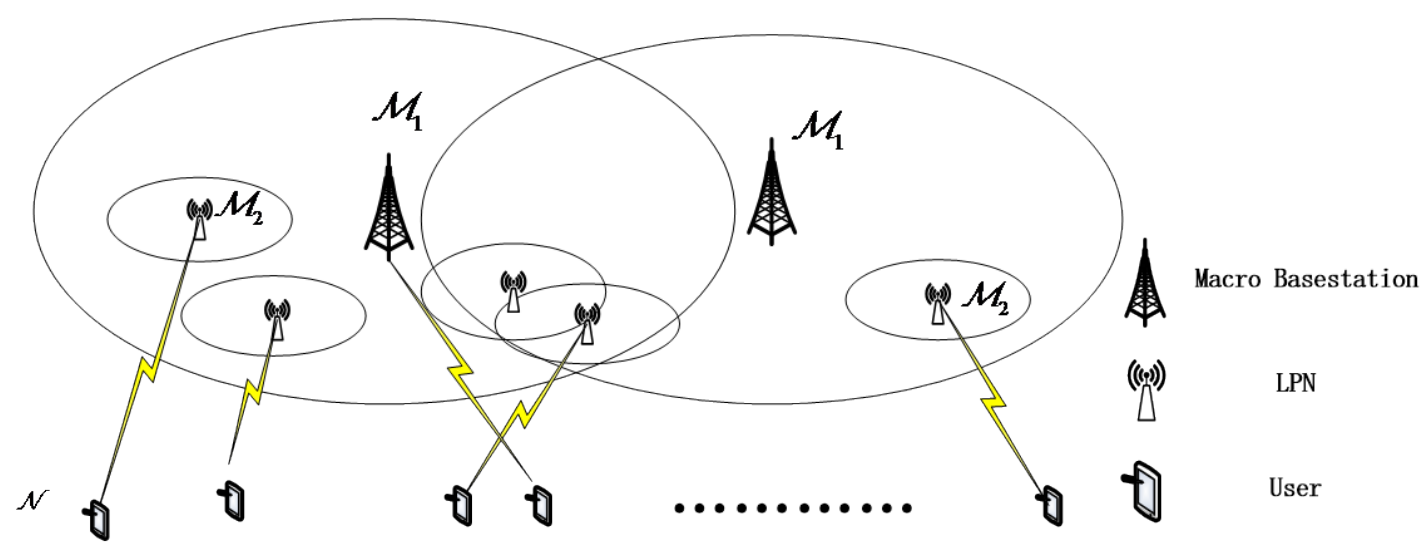

Fig. 1. system model

In wireless network, QoE assessment is a challenge, which draws a lot of interests from both academia and industry. The Mean Opinion Score(MOS) model is widely used to characterize QoE whose value can reflect users' opinions on service, which ranges from totally unacceptable to fully satisfied [3]. We integrate the MOS model into the QEEBA and classify the service as two class: $\mathrm{C}$ type service (e.g. voice service) and $\mathrm{S}$ type service (e.g. video service).

\subsection{The QoE function of C type service}

Traditionally, the main $C$ type service is voice service which usually measured by Perceptual Evaluation of Speech Quality (PESQ) recommended by International Telecommunication Union (ITU). However, it is still not suitable for dynamic real-time system because of the computationally complexity and requirement of the original speech signal. In [39], the QoE model for voice service is described as the function of transmission rate. We assuming that the transmission rate of C type service is $R_{c}$, the obtained utility of it will increase with the increasing transmission rate $R_{c}$ but the increasing rate will be slow down. Thus, the MOS function of the service can be denoted as:

$$
\operatorname{MOS}\left(R_{c}\right)=\log \left(R_{c}-R_{\min }+1\right) / \log \left(R_{\max }-R_{\min }+1\right)
$$


where, $R_{\max }$ and $R_{\min }$ are the maximum and the minimum requirement transmission rate of $\mathrm{C}$ type service, respectively. If the $R_{c}$ is higher than the $R_{\max }$, the $\operatorname{MOS}\left(R_{c}\right)=1$.

\subsection{The QoE function of S type service}

The $\mathrm{S}$ type service, such as video service which is a real-time service with adaptive coding or file download service which is non real-time service. As described in [40], these service can use S-shaped MOS function to reflect the satisfaction degree of users. The MOS value will not decrease to zero directly when the transmission rate is smaller than the required rate. We assume that the transmission rate of $\mathrm{S}$ type service is $R_{s}$. The obtained utility of service can increase with the increasing of transmission rate $R_{s}$ and the increase faster firstly and slow down lately. So the MOS function of the $\mathrm{S}$ type service is:

$$
\operatorname{MOS}(R)=\exp \left(\frac{\xi \times\left(R-R_{\max }\right)^{2}}{\left(R_{\max }-R_{\min }\right)^{2}}\right)
$$

where, $R_{\max }$ and $R_{\min }$ are the maximum and the minimum transmission rate of $\mathrm{S}$ type service respectively, $\xi$ is the constant value. If the $R_{s}$ is higher than the $R_{\max }$, the $\operatorname{MOS}\left(R_{s}\right)=1$.

\section{Problem Formulation}

The design of modern wireless communication system mainly focuses on achieving the desirable energy efficiency. A large body of research work has focused on this goal. Recent advances in video communications usher in new opportunities, but also bring about new challenges. In the traditional wireless network design, the network operator ignores the subjective opinions of wireless users, which would be more important in terms of wireless users. QoE can directly and efficient reflect the users subjective opinions. In this paper, we design a based joint allocation model that aims to attain the best trade off among energy efficiency and QoE.

\section{1 maximize the QoE-aware energy efficiency}

Our aim is to maximize the energy efficiency and the QoE, which would help to improve system performance and users experience. The unreasonable access selection and resource allocation can increase the interference and lead to the user experience decrease, so the optimal model is:

$$
\max F=\sum_{n=1}^{N} Q E E_{n}
$$

where, $Q E E_{n}$ is the QoE-aware energy efficiency of $n$th user and can be calculated by formulation (4), the system QoE-aware energy efficiency is the sum of all users.

$$
Q E E_{n}=\operatorname{MOS}\left(R_{n}\right) \times E E_{n}
$$

where, $\operatorname{MOS}(\bullet)$ is the QoE function of user, $E E_{n}$ and $R_{n}$ are the energy efficiency and transmission rate of $n$th user respectively, which can be calculated as follows.

(1) The transmission rate $R_{n}$

In wireless network, when a user accesses to a cell, the user would request the service and the cell support the channel and transmission power to transmit the service. Assuming that the 
transmission power of the cell which $n$th user in $m$ th cell is $p_{m n}$, if the $n$th user does not access to the $m$ th cell, the $p_{m n}=0$, the transmission rate of $n$th user is:

$$
R_{n}=\sum_{m=1}^{M} B \times \log _{2}\left(1+\gamma_{n}\right)
$$

where, $\gamma_{n}$ is signal to interference plus noise ratio (SINR), which can be denoted as:

$$
\gamma_{n}=\frac{h_{m n} p_{m n}}{\sum_{i=1}^{M} \sum_{j \in S_{\bar{n}}} h_{i n} p_{i j}+N_{0}}
$$

where, $h_{m n}$ is the channel gain between the $m$ th cell and $n$th user. $N_{0}$ is the additive white Gaussian noise (AWGN) power. $\sum_{i=1}^{M} \sum_{j \in S_{\bar{n}}} h_{i n} p_{i j}$ is the interference of $n$th user. Assuming the $n$th user access to the kth sub-channel, $S_{\tilde{n}}$ is a set consisting of the other users accessing to the same sub-channel as $n$th user. $S_{\tilde{n}}$ can be denoted by:

$$
S_{\tilde{n}}=\left\{j=1,2, . ., n-1, n+1, . ., N \mid a_{i j}^{k}=1, i=1,2, . ., M\right\}
$$

where, $k$ represents the sub-channel which the $n$th user accesses in. $a_{i j}^{k}=1$ means the $j$ th user access to the ith cell in $k$ th sub-channel.

(2) The energy efficiency $E E_{n}$

In wireless network, the energy efficiency of the $n$th user can be expressed as:

$$
E E_{n}=\frac{R_{n}}{\sum_{m=1}^{M} \xi_{m n} p_{m n}+p^{I}+\sum_{m=1}^{M} c_{m n} p_{m}^{D}} \times\left(1-P f_{n}\right)
$$

where, $\xi_{m n}$ is the inverse of the power amplifier efficiency of $n$th user in $m$ th cell, the second term, $p^{I}$, is the independent fixed power consumption by the users, and the last term represents the cell-related power consumption, where $p_{m}^{D}$ denotes the fixed power consumption of each sub-channel in $m$ th cell. $P f_{n}$ is the frame error rate, which is determined by bit error rate and coding schemes [23].

\subsection{Constraints}

In an optimization problem, constraints seriously affect the optimal solution of the objective function. Therefore, how to select constraints becomes an indispensable factor to solve the objective function. The access selection and power allocation is performed under following constraints:

- The accessing constraint: a sub-channel in each cell is allocated to at most one user at a time to avoid the needless interference among users in each sub-channel, which requires the following constraint:

$$
\sum_{n=1}^{N} a_{m n}^{k} \leq 1, k \in \mathcal{K}, m \in \mathcal{M}
$$

- The QoS constraint: the SINR at received terminal should meet the requirement to maintain its performance. Assuming that the recognition SINR of receiving set is $\gamma_{n \min }$, so we have the constraint: 


$$
\sum_{m=1}^{M} \frac{h_{m n} p_{m n}}{\sum_{i=1}^{M} \sum_{j \in S_{\bar{n}}} h_{i n} p_{i j}+N_{0}} \geq \gamma_{n \min }, \forall n \in \mathcal{N}
$$

- The power constraint: In practice, the transmission power available in $m$ th cell, denoted by $p_{\text {max }}$, is limited for downlink transmission. $p_{\text {max }}$ not only controls the out-cell interference, but also restricts the power amplifier to linear region which corresponds to the constant amplifier efficiency. Thus, the total transmission power in the $m$ th cell should satisfy:

$$
\sum_{n=1}^{N} p_{m n}<p_{\max }, \forall m \in \mathcal{M}
$$

\section{Joint Access selection and power allocation algorithm}

Above all, our target is to maximize the QoE and energy efficiency, thus, the problem can be formulated as the following optimization model:

$$
\left\{\begin{array}{l}
\max F=\sum_{n=1}^{N} Q E E_{n} \\
s t\left\{\begin{array}{l}
\sum_{n=1}^{N} a_{m n}^{k} \leq 1, k \in \mathcal{K}, m \in \mathcal{M} \\
\sum_{m=1}^{M} \frac{h_{m n} p_{m n}}{\sum_{i=1}^{M} \sum_{j \in S_{\bar{n}}} h_{i n} p_{i j}+N_{0}} \geq \gamma_{n \min }, \forall n \in \mathcal{N} \\
\sum_{n=1}^{N} p_{m n}<p_{\max }, \forall m \in \mathcal{M}
\end{array}\right.
\end{array}\right.
$$

Considering that the feasible solution space of this optimization problem is huge, it is difficult to find the optimal solution using enumeration method. Therefore, the proposed algorithm should have a fast convergence speed to get the optimal solution. Some optimal algorithms proposed in [41-43] cannot solve this mixed integer nonlinear programming problem. We also know that the immune algorithm could solve the optimization problem, however, its convergence speed is slow and it may fall into local optimal trap easily. So, in this paper, a novel algorithm named CCIA is proposed. Because of the strong ergodicity and randomness of chaotic system, the antibody population can randomly distribute in solution space and the CCIA can get the optimal value exactly. The key methods can be summarized as follows:

(1) Initialization

Set the generation counter $t=1$ and the number of initial antibody population is $Q$. Let $p_{n}^{q}(t)$ and $b_{n}^{q}(t)$ denote the transmission power and access selection option of the $n$th user respectively. If the nth user accesses to the $m$ th cell, the $p_{n}^{q}(t)$ would equal to the $p_{m n}$, otherwise, the $p_{n}^{q}(t)=0$. The $b_{n}^{q}(t)$ can be given in: 


$$
b_{n}^{q}(t)= \begin{cases}k+K \times(m-1), & a_{m n}^{k}=1 \\ 0, & a_{m n}=0\end{cases}
$$

Let $A^{q}=\left\{P^{q}, B^{q}\right\}=\left\{\left(p_{1}^{q}, b_{1}^{q}\right), \ldots,\left(p_{n}^{q}, b_{n}^{q}\right), \ldots,\left(p_{N}^{q}, b_{N}^{q}\right)\right\}$ denote the antibody. It is obviously that $A^{q}$ is a two-dimension array with $N$ elements. To initialize the antibody, we first use the Roulette method to get the network each user accesses, and the random value in Roulette is get from the chaotic system, then we initialize the transmission power according to the user accesses method. In this paper, we derive the chaotic values in the chaotic system and map these values into the $\left[0, p_{\max }\right]$, and we get the value in this sequence as the transmission power, randomly. In which, $p_{\max }$ is a variable value. If the user accesses to the $m$ th network, $p_{\max }=p_{\text {m max }}$, otherwise, $p_{\max }=0$.

(2) Chaotic system

The chaotic sequence is generated by the four-dimensional chaotic system [44]. Then used in initialization process and mutation process. The four-dimensional chaotic system can be generated by:

$$
\left\{\begin{array}{l}
s^{\prime}=a(-s+t)+t u v \\
t^{\prime}=b(s+t)-s u v \\
u^{\prime}=c t-v+d s t v \\
v^{\prime}=-e v+s t u
\end{array}\right.
$$

Where, the $a, b, c, d, e$ are parameters of four-dimensional chaotic system. The sequence $s, t, u, v$ are the chaotic sequence, which can be used to get random value.

(3) Clone and Mutation

Clone and mutation methods are same as the clone immune algorithm in [21]. The mutation probability is got from chaotic sequence $s$.

The obtained fitness function represents the maximum joint QoE-aware Energy Efficiency and the corresponding antibodies represent the optimal power and access selection solutions. The detailed description is displayed in Fig. 2.

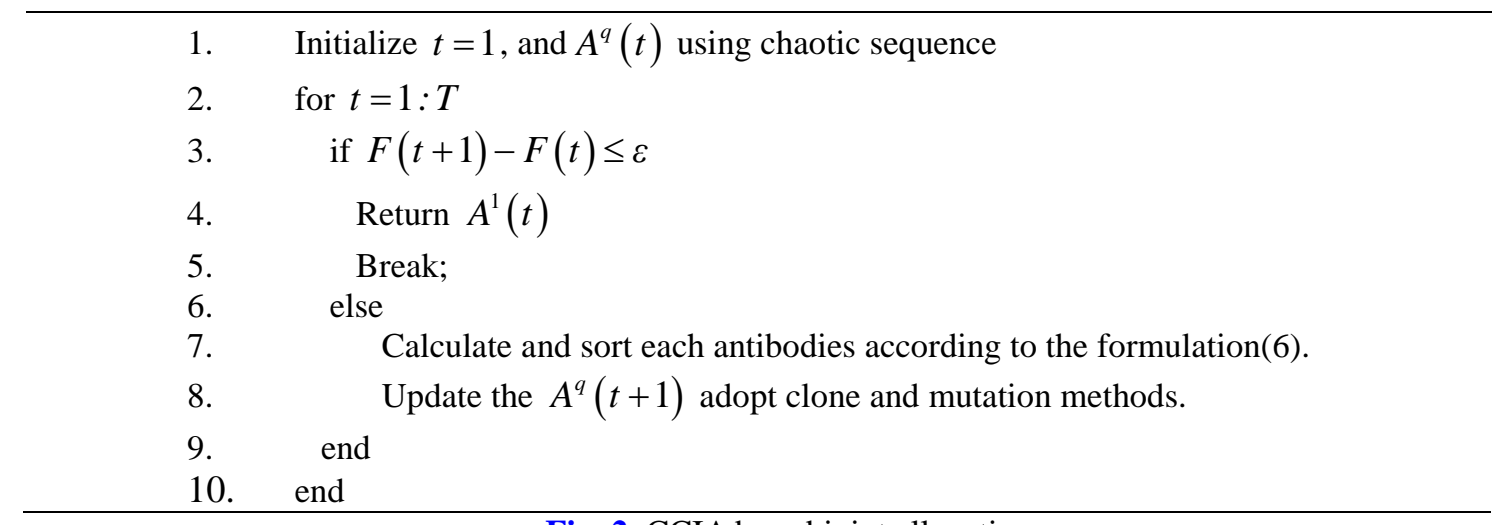

Fig. 2. CCIA based joint allocation.

(4) Complexity Analysis

Firstly, the initialization using chaotic system is only one operation each iteration in proposed method. The object value calculation of each iteration need $O(M N Q)$, The object 
value sorting of each iteration need $O\left(Q^{2}\right)$. The clone of each iteration need $O(L), L$ is the number of elite antibody. The mutation of each iteration need $O(N)$. Therefore, the complexity is $O\left(T\left(M N Q+Q^{2}\right)\right)$.

\section{Simulation Experiment and Result Analysis}

The previous section is based on an analysis of the optimization problem in theory. In this section, the accuracy of the analysis is evaluated experimentally. To solve the optimization problem, we choose the chaotic clone immune algorithm. Here, the performance of the proposed algorithm is compared with other algorithms.

All the experiments are performed in the same simulation scenario shown in Fig. 1. The coverage radius of the macro-cell is $500 \mathrm{~m}$, and LPN is $10 \mathrm{~m}$. Each user is randomly distributed in the network. Additionally, the large-scale fading exponent is given by $\alpha$.The experiment parameters are showed in Table 1 [44,45].

Table 1. Simulation Parameters

\begin{tabular}{ll}
\hline Parameter & Value \\
\hline Sub-channel Bandwidth(B) & $200 \mathrm{KHz}$ \\
Number of sub-channels(K) & 30 \\
The AWGN power spectral density(N0) & $-136 \mathrm{dBm}$ \\
Maximum transmit power of macro base station & $30 \mathrm{w}$ \\
Maximum transmit power of LPN & $3 \mathrm{w}$ \\
The exponent of large-scale fading( $\alpha)$ & 2 \\
Circuit Power of macro base station in each sub-channel & $0.1 \mathrm{w}$ \\
Circuit Power of LPN in each sub-channel & $0.04 \mathrm{w}$ \\
Independent fixed power consumption by the user & $0.1 \mathrm{~W}$ \\
Inverse of the power amplifier efficiency & 0.38 \\
The covering radio of macro-cell network & $500 \mathrm{~m}$ \\
The covering radio of LPN & $10 \mathrm{~m}$ \\
Initial antibody population Q & 100 \\
Maximum number of generations T & 100 \\
\hline
\end{tabular}

\subsection{Performance of chaotic clone immune algorithm}

The paper uses CCIA to solve the optimal problem proposed in Sec.3. This algorithm is named QEEBA. In order to verify the convergence and convergence speed of QEEBA, we compare the QEEBA with immune algorithm (IA). Fig. 3 shows the performance of QoE-aware energy efficiency versus the generations of QEEBA and IA calculated. It can be seen in Fig. 3 that, in the same iteration, the result of QEEBA is superior to the IA which means that the convergence speed of QEEBA is faster than IA. It is also obvious that the QEEBA and IA fall into the same local optimization trap when in about 15 and 25, respectively, and the QEEBA can avoid the local optimization trap in about 30 and get the higher optimal value than IA. 


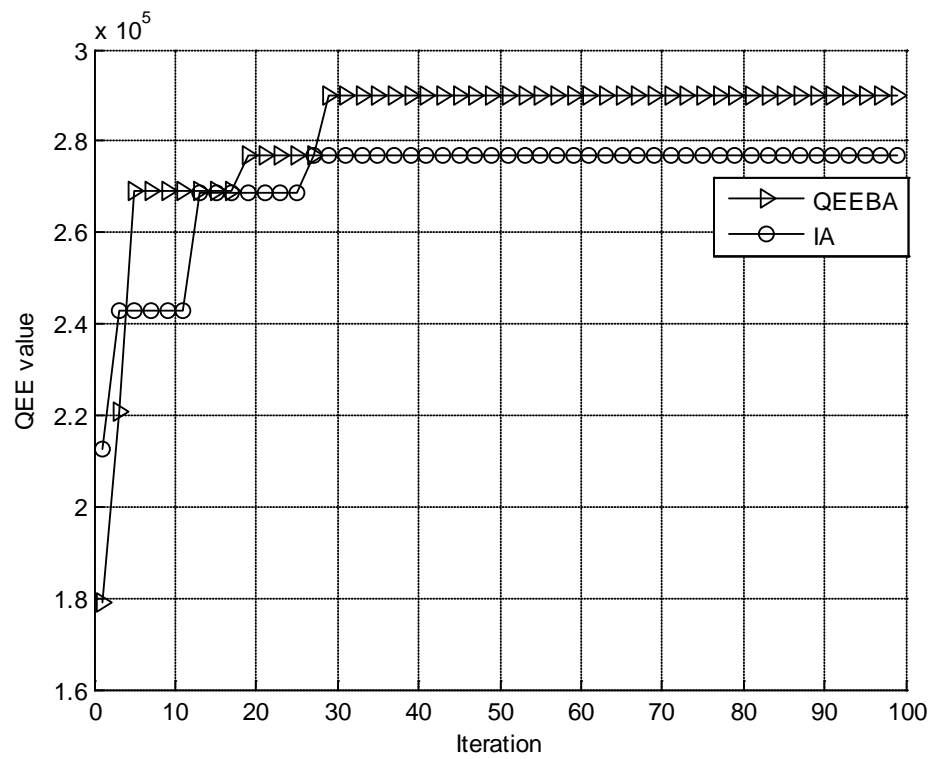

Fig. 3. optimal values iteration.

\subsection{Energy efficiency versus number of users}

Fig. 4 shows the energy efficiency of the system using four different algorithms. As shown in the figure, the energy efficiency is increasing with the decelerating growth rate, while the user number varies from 10 to 70 . It is because that the resource in heterogeneous network is limited, and the energy efficiency of each user will decrease with the increasing of user number. It is obvious that the EEBA has the highest energy efficiency in four algorithms because its object is the maximization of energy efficiency. The QEEBA is only worse than EEBA and superior than other two algorithms. The reason is that the QEEBA also consider the energy efficiency, it would be make a tradeoff between the utility of users and energy efficiency.

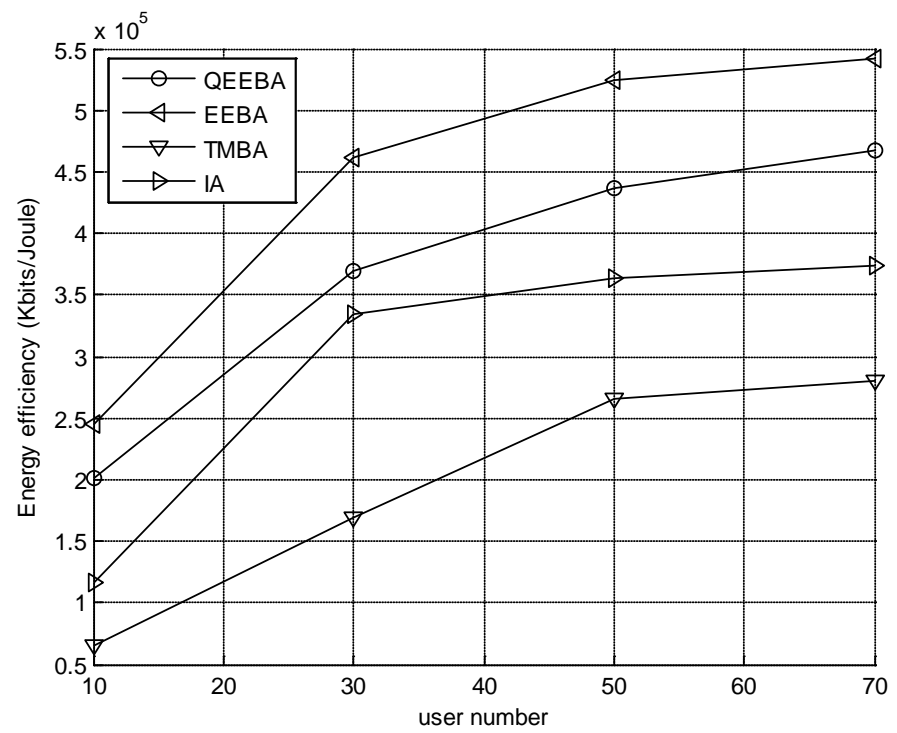

Fig. 4. Energy efficiency of system 
Fig. 5 shows the energy efficiency of the macro network and LPNs. It is obvious that the energy efficiency of LPN in EEBA is much higher than in QEEBA while the energy efficiency of macro-cell in EEBA is lower than in QEEBA. The EEBA pursue the maximization of energy efficiency and much users would access to LPN to improve the personal energy efficiency, which would cause the user QoE decreases. However, the QEEBA would sacrifice the energy efficiency to improve the user QoE which is also be demonstrated in Fig. 6 and Fig. 8.

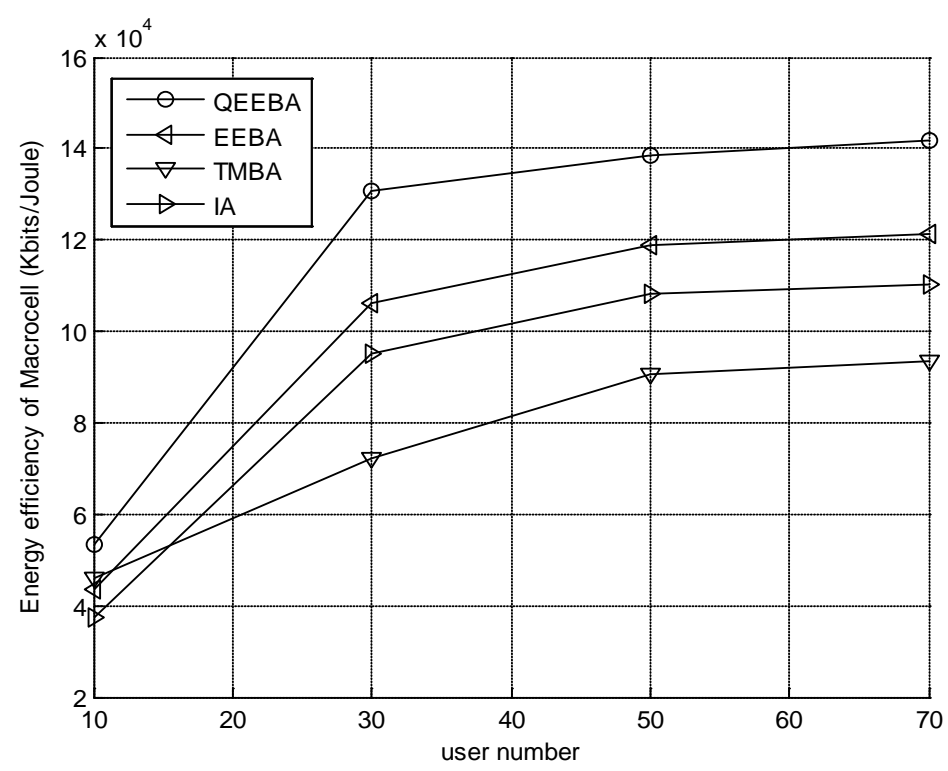

(a)

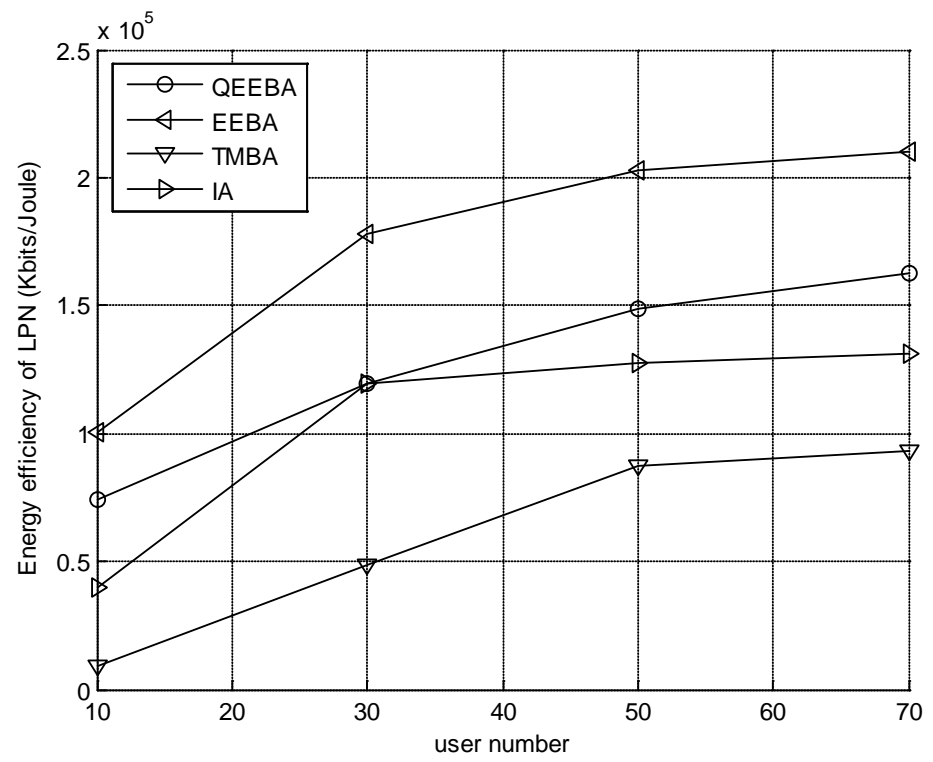

(b)

Fig. 5. Energy efficiency in different network. (a) Macro-cell network (b) LPN network 


\subsection{The QoE analysis}

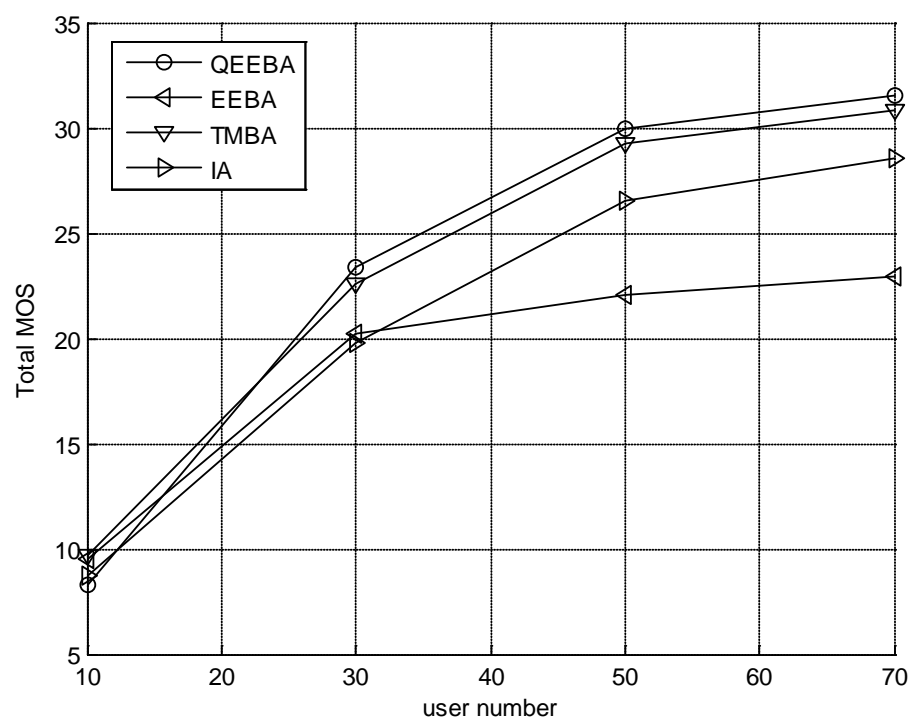

Fig. 6. Average MOS of users

Fig. 6 illustrates the QoE of users, as we investigate in Sec.2, the QoE of users is measured by MOS. So, we show the average MOS value in Fig. 6. It is obviously that the MOS in QEEBA is far higher than in EEBA because the QoE is considered in QEEBA. We can observe that the MOS in QEEBA is close to it in TMBA. The reason is that the QoE function is related to the transmission rate, the TMBA also can improve the QoE of users. While the QEEBA directly consider the QoE of users, so it can achieve the close performance to TMBA though the energy efficiency is also considered in QEEBA.

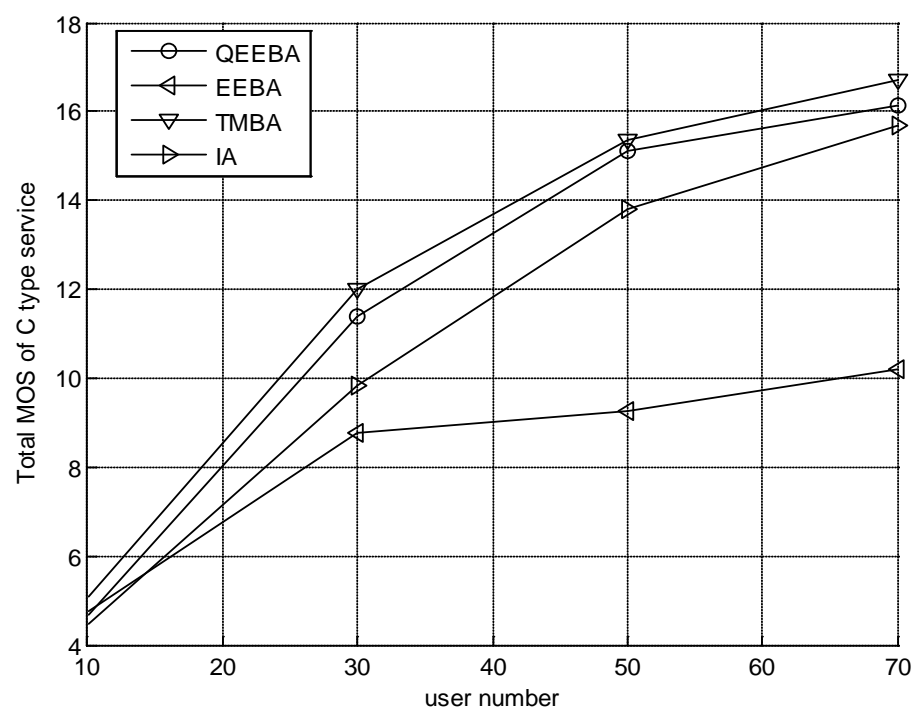

(a) 


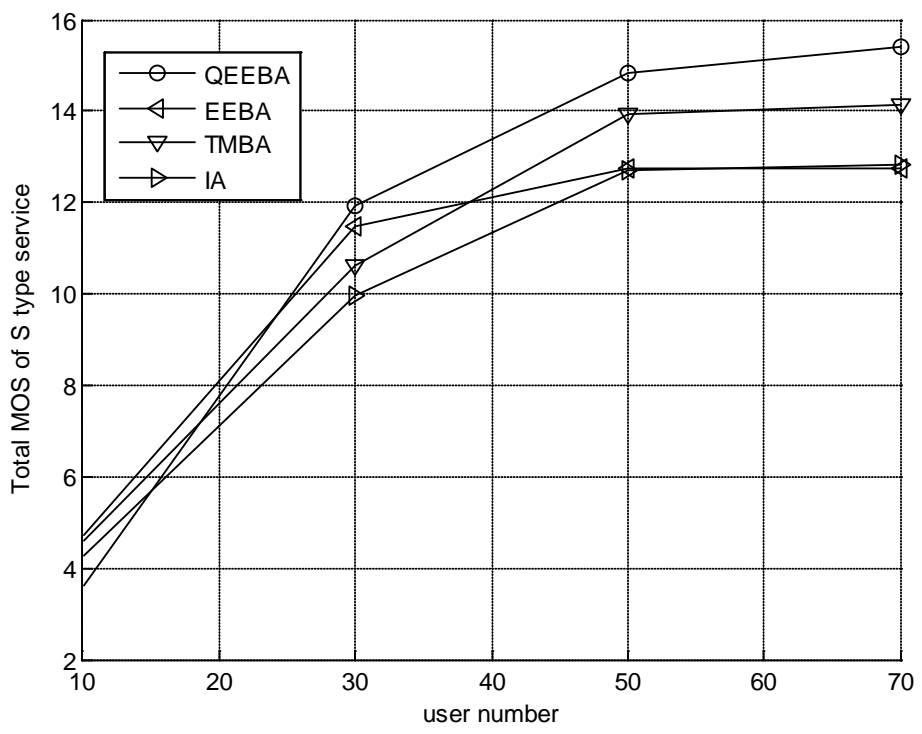

(b)

Fig. 7. The average MOS of users with different type service. (a) $\mathrm{C}$ type service (b) $\mathrm{S}$ type service

Fig. 7 shows the QoE performance of different type service in four algorithms. The Fig. 6 further demonstrate the reason of the near QoE performance between TMBA and QEEBA. As we can discover, The QEEBA would be fairer to $S$ type service and $C$ type service than the TMBA because it directly consider the QoE of different service.

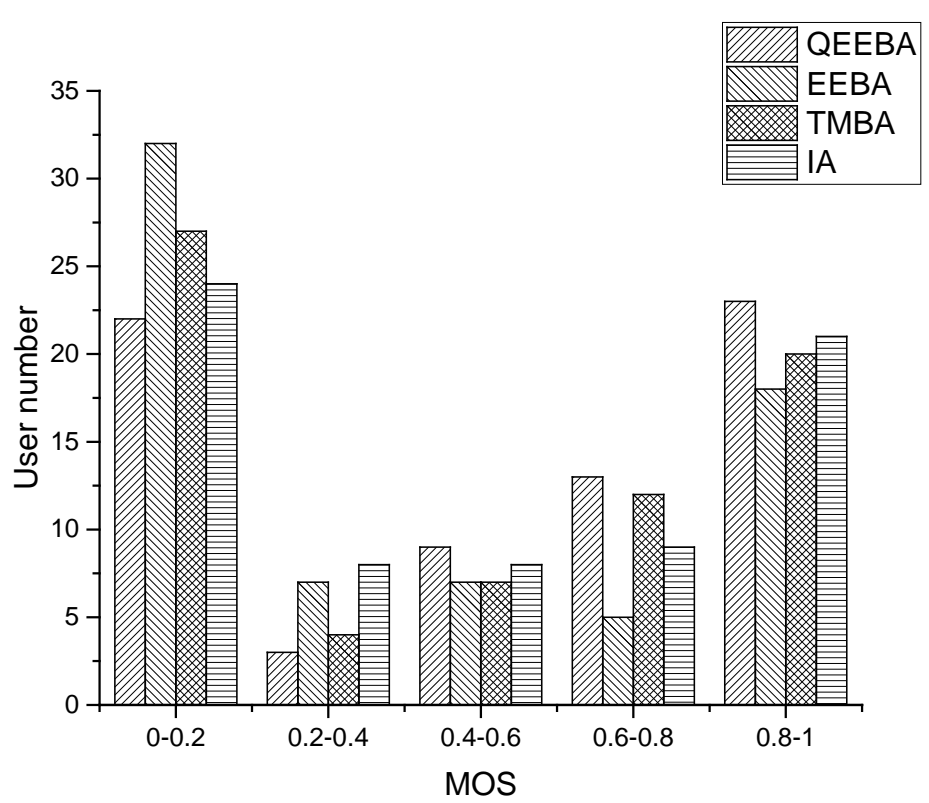

Fig. 8. The number of users versus the MOS 
As shown in Fig. 8, the QEEBA has the largest number of user with good QoE ( $M O S \in(0.6,1]$ ) in four algorithms and the TMBA takes the second place. While the number of user with bad QoE is the lowest MOS $\in[0,0.4]$ in QEEBA. The reason is that the users would improve their personal QoE to promote the total QoE in QEEBA and TMBA; however, the EEBA pursues the energy efficiency excessively and ignore the QoE of users. The IA takes second place because it can fall into local optimization trap and not get the optimal value.

\section{Conclusion}

In this paper, a joint power and bandwidth allocation algorithm based on QoE-aware energy efficiency of users was proposed. The joint allocation problem was transformed into the optimization problem and the chaotic clone immune algorithm was adopted to solve it. Simulation results demonstrate that the proposed algorithm can improve the QoE and improve the energy efficiency. There is also an important issue unaddressed in this paper and left for future work. Since the the object function is not continuously differentiable, the proposed problem can not translate to convex optimization problem and is solved by a heuristic approach, The challenge is to establish a new MOS model whose deviation from this proposed MOS is negligible and need further investigation.

\section{References}

[1] A. Damnjanovic, J. Montojo, Y. Wei, T. Ji, T. Luo, M. Vajapeyam, T. Yoo, O. Song and D. Malladi, "A survey on 3GPP heterogeneous networks," IEEE Wireless Communications, vol. 18, no. 3, pp. 10-21, June, 2011. Article (CrossRef Link).

[2] H. Zhang, C. Jiang, N. C. Beaulieu, X. Chu, X. Wen and M. Tao, "Resource Allocation in Spectrum-Sharing OFDMA Femtocells with Heterogeneous Services," IEEE Transactions on Communications, vol. 62, no. 7, pp. 2366-2377, June, 2014. Article (CrossRef Link).

[3] J. Dai and S. Wang, "QoE-driven resource allocation method for cognitive radio networks," in Proc. of 2016 in IEEE International Conference on Communications (ICC), Kuala Lumpur, pp. 1-6, July, 2016. Article (CrossRef Link).

[4] H. Shao et al., "Joint Optimization of Quality of Experience and Power Consumption in OFDMA Multicell Networks," IEEE Communications Letters, vol. 20, no. 2, pp. 380-383, Feb. 2016. Article (CrossRef Link).

[5] Y. S. Soh, T. Q. S. Quek, M. Kountouris and H. Shin, "Energy Efficient Heterogeneous Cellular Networks,” IEEE Journal on Selected Areas in Communications, vol. 31, no. 5, pp. 840-850, May, 2013. Article (CrossRef Link).

[6] B. S. Rong, F. R. Yu, and G. Senarath. "Interference-Aware Energy-Efficient Resource Allocation for OFDMA-Based Heterogeneous Networks with Incomplete Channel State Information," in IEEE Transactions on Vehicular Technology, vol. 64, no. 3, pp. 6081-6085, Mar. 2014. Article (CrossRef Link).

[7] C. Han, T. Harrold, S. Armour, J. Krikidis, S. Videv, P. M. Grant, H. Haas, J. S. Thompson, I. Ku, C. X. Wang, T. A. Le, M. K. Nakhai, J. Zhang and L. Hanzo, "Green radio: radio techniques to enable energy-efficient wireless networks," in IEEE Communications Magazine, vol. 49, no. 6, pp. 46-54, June 2011. Article (CrossRef Link).

[8] T. Chen, Y. Yang, H. Zhang, H. Kim and K. Horneman, "Network energy saving technologies for green wireless access networks,” in IEEE Wireless Communications, vol. 18, no. 5, pp: 30-38, May. 2011. Article (CrossRef Link).

[9] A. Kiran, B. Singh, and R. Khanna, "Particle swarm optimization based network selection in heterogeneous wireless environment," in Optik - International Journal for Light and Electron Optics, vol. 125, no.1,pp:214-129, Jan.2014. Article (CrossRef Link). 
[10] K. Pavlos, A. Rouskas, and M. Anagnostou. "Utility-based RAT selection optimization in heterogeneous wireless networks," in Pervasive \& Mobile Computing, vol.12, no.10, pp. 92-111, June 2014. Article (CrossRef Link).

[11] Y. Kwon, T. Hwang and X. Wang, "Energy-Efficient Transmit Power Control for Multi-tier MIMO HetNets," in IEEE Journal on Selected Areas in Communications, vol. 33, no. 10, pp. 2070-2086, Oct. 2015. Article (CrossRef Link).

[12] T. Mao, G. Feng, L. Liang, S. Qin and B. Wu, "Distributed Energy-Efficient Power Control for Macro-Femto Networks," in IEEE Transactions on Vehicular Technology, vol. 65, no. 2, pp. 718-731, Feb. 2016. Article (CrossRef Link).

[13] C. Jiang, Y. Chen, K. J. R. Liu and Y. Ren, "Renewal-theoretical dynamic spectrum access in cognitive radio network with unknown primary behavior," in IEEE Journal on Selected Areas in Communications, vol. 31, no. 3, pp. 406-416, Mar. 2013. Article (CrossRef Link).

[14] X. Kang, R. Zhang and M. Motani, "Price-Based Resource Allocation for Spectrum-Sharing Femtocell Networks: A Stackelberg Game Approach," in IEEE Journal on Selected Areas in Communications, vol. 30, no. 3, pp. 538-549, Apr. 2012. Article (CrossRef Link).

[15] R. Chai, H. Zhang and Q. Chen, "Throughput maximization based joint power and bandwidth allocation for UMTS/WLAN integrated network," in 2013 8th International Conference on Communications and Networking in China (CHINACOM), pp. 246-251, Aug 14-16, 2013. Article (CrossRef Link).

[16] P. Luong, T. M. Nguyen, L. B. Le and N. D. ðào, "Admission control design for integrated WLAN and OFDMA-based cellular networks," in Proc. of 2014 IEEE Wireless Communications and Networking Conference (WCNC), pp. 1386-1391, Apr 6-9, 2014. Article (CrossRef Link).

[17] T. Maksymyuk, M. Kyryk and M. Jo, "Comprehensive Spectrum Management for Heterogeneous Networks in LTE-U," IEEE Wireless Communications, vol. 23, no. 6, pp. 8-15, Dec. 2016. Article (CrossRef Link).

[18] N. Ul Hasan, W. Ejaz, N. Ejaz, H. S. Kim, A. Anpalagan and M. Jo, "Network Selection and Channel Allocation for Spectrum Sharing in 5G Heterogeneous Networks," IEEE Access, vol. 4, pp. 980-992, Feb. 2016. Article (CrossRef Link).

[19] G. Huang, Z. Lin, D. Tang and J. Qin, "QoS-driven jointly optimal power and bandwidth allocation for heterogeneous wireless networks," Electronics Letters, vol. 51, no. 1, pp. 122-124, Jan. 2015. Article (CrossRef Link).

[20] C. A. Chan, W. Li, S. Bian, C. Lin, A. F. Gygax, C. Leckie, M. Yan and K. Hinton. "Assessing network energy consumption of mobile applications," IEEE Communications Magazine, vol. 53, no. 11, pp. 182-191, Nov. 2015. Article (CrossRef Link).

[21] M. Elhoseny, X. Yuan, Z. Yu, C. Mao, H. K. El-Minir and A. M. Riad, "Balancing Energy Consumption in Heterogeneous Wireless Sensor Networks Using Genetic Algorithm," IEEE Communications Letters, vol. 19, no. 12, pp. 2194-2197, Dec. 2015. Article (CrossRef Link).

[22] Q. Wu, G. Y. Li, W. Chen, D. W. K. Ng and R. Schober. “An Overview of Sustainable Green 5G Networks,” IEEE Wireless Communications, Oct. 2016. Article (CrossRef Link).

[23] W. Y. Wang, X. Wang and A. A. Nilsson, "Energy-efficient bandwidth allocation in wireless networks: algorithms, analysis, and simulations," in IEEE Transactions on Wireless Communications, vol. 5, no. 5, pp. 1103-1114, May 2006. Article (CrossRef Link).

[24] S. Zhang, Q. Wu, S. Xu and G. Y. Li, "Fundamental Green Tradeoffs: Progresses, Challenges, and Impacts on 5G Networks," IEEE Communications Surveys \& Tutorials, vol. 19, no. 1, pp. 33-56, July. 2017. Article (CrossRef Link).

[25] Q. Wu and W. Chen, "User-centric energy-efficient resource allocation for wireless powered communications," in Proc. of 2015 International Conference on Wireless Communications \& Signal Processing (WCSP), pp. 1-6, Oct 15-17, 2015. Article (CrossRef Link).

[26] G. Y. Li, Z. Xu, G. Xiong, C. Yang, S. Zhang, Y. Chen and S. Xu. "Energy-efficient wireless communications: Tutorial, survey, and open issues," IEEE Wireless Communications, vol. 18, no. 6, pp. 28-35, Dec. 2011. Article (CrossRef Link).

[27] J. B. Rao and A. O. Fapojuwo, "A survey of energy efficient resource management techniques for multicell cellular networks,” IEEE Communications Surveys \& Tutorial., vol. 16, no. 1, pp. 
154-180, May. 2014. Article (CrossRef Link).

[28] S. Ji, L. Tang, S. Du and S. Li, "Energy efficiency maximization based joint power and bandwidth allocation for heterogeneous network," in Proc. of 2016 12th International Conference on Natural Computation, Fuzzy Systems and Knowledge Discovery (ICNC-FSKD), pp. 2019-2023, Aug 13-15, 2016. Article (CrossRef Link).

[29] G. Lim, C. Xiong, L. J. Cimini and G. Y. Li, "Energy-Efficient Resource Allocation for OFDMA-Based Multi-RAT Networks," IEEE Transactions on Wireless Communications, vol. 13, no. 5, pp. 2696-2705, May 2014. Article (CrossRef Link).

[30] Q. Wu, W. Chen, M. Tao, J. Li, H. Tang and J. Wu, "Resource Allocation for Joint Transmitter and Receiver Energy Efficiency Maximization in Downlink OFDMA Systems," IEEE Transactions on Communications, vol. 63, no. 2, pp. 416-430, Feb. 2015. Article (CrossRef Link).

[31] P. Luong, T. M. Nguyen, L. B. Le, N. D. Đào and E. Hossain, "Energy-Efficient WiFi Offloading and Network Management in Heterogeneous Wireless Networks," IEEE Access, vol. 4, pp. 10210-10227, 2016. Article (CrossRef Link).

[32] Y. S. Soh, T. Q. S. Quek, M. Kountouris and H. Shin, "Energy Efficient Heterogeneous Cellular Networks," IEEE Journal on Selected Areas in Communications, vol. 31, no. 5, pp. 840-850, May 2013. Article (CrossRef Link).

[33] Y. Xu, R. Q. Hu, Y. Qian and T. Znati, "Video Quality-Based Spectral and Energy Efficient Mobile Association in Heterogeneous Wireless Networks," IEEE Transactions on Communications, vol. 64, no. 2, pp. 805-817, Feb. 2016. Article (CrossRef Link).

[34] H. Pervaiz, L. Musavian and Q. Ni, "Joint user association and energy-efficient resource allocation with minimum-rate constraints in two-tier HetNets," in Proc. of 2013 IEEE 24th Annual International Symposium on Personal, Indoor, and Mobile Radio Communications (PIMRC), pp. 1634-1639, Sept 8-11, 2013. Article (CrossRef Link).

[35] Q. Wu, W. Chen and J. Li, "Wireless Powered Communications With Initial Energy: QoS Guaranteed Energy-Efficient Resource Allocation," IEEE Communications Letters, vol. 19, no. 12, pp. 2278-2281, Dec. 2015. Article (CrossRef Link).

[36] K. U. R. Laghari and K. Connelly, "Toward total quality of experience: A QoE model in a communication ecosystem," IEEE Communications Magazine, vol. 50, no. 4, pp. 58-65, Apr. 2012. Article (CrossRef Link).

[37] Y. Y. Zhang, H. Long, Y. X. Peng, A. V. Vasilakos, and W. B. Wang. "QoE and energy efficiency aware resource allocation for OFDM systems in group mobility environments," International Journal of Communication Systems, vol. 27, no. 12, pp. 3526-3544. Dec. 2014. Article (CrossRef Link).

[38] B. Li, S. Li, C. Xing, Z. Fei and J. Kuang, "A QoE-based OFDM resource allocation scheme for energy efficiency and quality guarantee in multiuser-multiservice system," in Proc. of 2012 IEEE Globecom Workshops, pp. 1293-1297, Dec 3-7, 2012. Article (CrossRef Link).

[39] S. Thakolsri, W. Kellerer and E. Steinbach, "QoE-Based Cross-Layer Optimization of Wireless Video with Unperceivable Temporal Video Quality Fluctuation," in Proc. of 2011 IEEE International Conference on Communications (ICC), pp. 1-6, June 5-9, 2011. Article (CrossRef Link).

[40] Q. T. Nguyen-Vuong, Y. Ghamri-Doudane and N. Agoulmine, "On utility models for access network selection in wireless heterogeneous networks," in Proc. of NOMS 2008 - 2008 IEEE Network Operations and Management Symposium, pp. 144-151, Apr 7-11, 2008. Article (CrossRef Link).

[41] A. Karadimce, R. Malekian, and A. H. Abdullah. "Intelligent Delivery of Multimedia Content in Mobile Environment," Applied Mathematics \& Information Sciences, vol. 7, no. 6, pp. 2305-2310, Nov. 2013. Article (CrossRef Link).

[42] L. Wang, J. Shao, Y. Q. Zhong, W. S. Zhao and R. Malekian, "Modeling Based on Elman Wavelet Neural Network for Class-D Power Amplifiers," Applied Mathematics \& Information Science, vol. 7, no. 6, pp. 2445-2453, Nov. 2013. Article (CrossRef Link).

[43] R. Malekian, D. C. Bogatinoska, A. Karadimce, N. Ye, J. Trengoska and W. A. Nyako, "A Novel Smart ECO model for Energy Consumption Optimization," Elektronika Ir Elektrotechnika, vol. 21, 
no. 6, pp. 75-80, 2015. Article (CrossRef Link).

[44] L. Tang, W. Li, J. Sheng and B. Fan. "A Chaos Genetic Algorithm Based Access Selection in Heterogeneous Wireless Networks,” Acta Electronica Sinica, vol. 42, no. 8, pp:1564-1570. Aug, 2014. Article (CrossRef Link).

[45] G. Lim, C. Xiong, L. J. Cimini and G. Y. Li, "Energy-Efficient Resource Allocation for OFDMA-Based Multi-RAT Networks," IEEE Transactions on Wireless Communications, vol. 13, no. 5, pp. 2696-2705, May, 2014. Article (CrossRef Link).
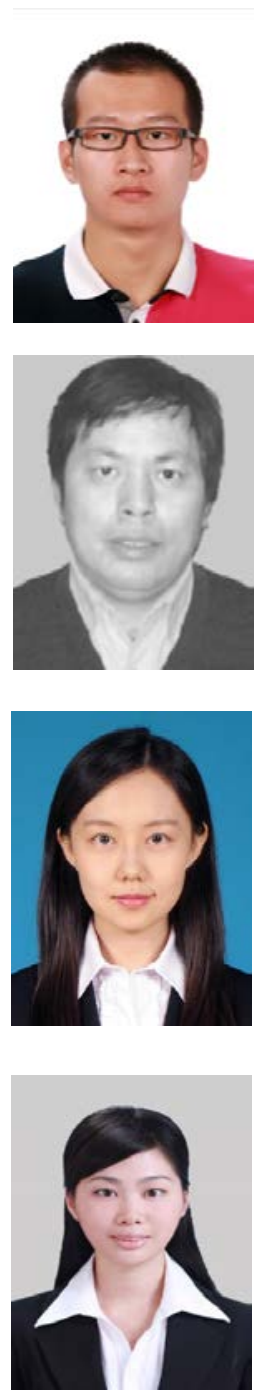

Shiyu Ji received the B.S. degree in 2013 and now is a candidate for Ph.D. degree in Electrical Information Technology of North China Electric Power University, Beijing, China. His research interests include wireless networks and communication technology in power system.

Liangrui Tang received the Ph.D. degree in Communication and Information System from Beijing University of Posts and Telecommunications. Now, he is a Professor in State Key Laboratory of Alternate Electrical Power System with Renewable Energy Sources of North China Electric Power University, focusing on the research of communication in power system, wireless communications and optical network communication

Chen Xu received the B.E. degree from Beijing University of Posts and Telecommunications, in 2010, and the Ph.D. degree from Peking University, in 2015. She is now a lecturer in School of Electrical and Electronic Engineering, North China Electric Power University, China. Her research interests mainly include wireless resource allocation and management, game theory, optimization theory, heterogeneous networks, and smart grid communication.

Shimo Du received the M.S. degree in Electronic and Information Engineering from North China Electric Power University, Beijing, China, in 2014. And now, she is currently an engineer in China mobile communication corporation Hangzhou branch. Her research interests include congestion control, network traffic analysis and communication technology in power system. 


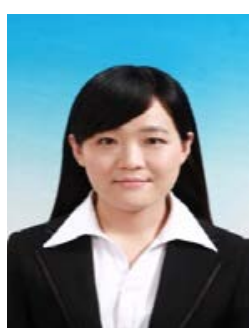

Jiajia Zhu received the B.Eng. degree in Electronic Information Engineering from North China Electric Power University in July 2014. She is currently pursuing her Ph.D. degree in Electrical Engineering of North China Electric Power University. Her research interests cover interference management and resource allocation in heterogeneous networks.8

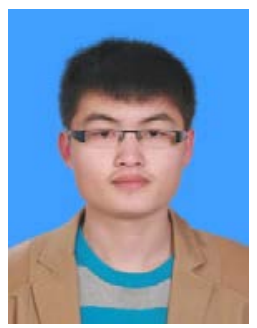

Hailin Hu received the B.Eng. degree in Telecommunications Engineering from North China Electric Power University in July 2016. He is currently pursuing his Ph.D. degree in Electrical Engineering of North China Electric Power University. His research interests cover resource allocation in heterogeneous networks. 